\title{
Spectrum Sensing in Multi-user Cognitive Radio Networks
}

\author{
Yunxiao Zu, Tao Chen, Junjie Ding, Bin Hou, Dongjing Hu \\ School of Electronic Engineering, Beijing University of Posts and Telecommunication, Beijing, China \\ Email: zuyx@bupt.edu.cn
}

Received April, 2013

\begin{abstract}
Spectrum sensing is studied for multi-user cognitive radio networks in this paper. An improved spectrum sensing method----energy-cooperative detection method is developed, which combines the double threshold energy detecting method with the "OR" fusion decision rule. The simulation is done for both AWGN channel and Rayleigh channel, and the advantages of the improved method are verified.
\end{abstract}

Keywords: Cognitive Radio; Spectrum Detection; Energy Detection; Double-Threshold; Fusion Decision

\section{Introduction}

Spectrum sensing is an important technology of cognitive radio networks. There are many methods about spectrum detection [1-5]. The current main detection methods can be divided into single-node detection and cooperative detection. The single-node detection contains matched filter detection, energy detection and cyclostationary detection. These detection methods have their individual different advantages and disadvantages. The matched filter detection is fast, but the priori information is needed. The energy detection is simple and does not require the priori information, but it is easy to be affected by noise and needs a long time. The cyclostationary detection can distinguish noise from signal, but the computation complexity is high and the structure is complex.

The cooperative detection means that many users detect cooperatively in order to improve the detection performance. It involves two key technologies which are node selection and fusion decision rule. In cooperative detection every cognitive user worked in local is an individual. The overall detection performance is affected by the number, location and behavior of these cognitive users. About the node selection, all nodes are involved or some nodes with high reliability to be participated. In traditional cooperative detection algorithms all users are assumed to be involved, but literature [6] showed that all users are involved in the detection does not always reach the performance optimization, and the channel overheads are increased. Instead, the optimal detection performance can be achieved by selecting some local users with higher SNR to participate the cooperative detection. There are many decision rules [7]. The selection of the decision rule has a significant impact on detection performance, which is the focus of our work.
According to the characteristics of the multi-user cognitive radio networks, by analyzing the dual threshold energy detection method and the cooperative detection method, a spectrum detection method used for the multiuser cognitive radio networks is developed in this paper, which combines the above two detection methods and is called cooperative-energy detection method.

The rest of this paper is organized as follows. In Section II the energy-cooperative detection method is developed. In section III the simulation results and analysis are shown. The conclusions are given in Section IV.

\section{Energy-cooperative Detection Method}

\subsection{The Cooperative Detection Model}

As mentioned above, the two key technologies of cooperative detection are nodes selection and fusion decision rule. In this paper we only study the fusion decision rule which has more impact on the detection performance, which is all users participate operative detection.

In terms of the data form transmitted to the decision center from each cognitive user, the fusion decision rule can be divided into two types, one is the soft-decision and another is the hard-decision. In soft-decision, what the cognitive user transmits is the local detection analog signal. While in the hard-decision, the cognitive user makes decision in local, and transmits the information which shows whether the primary user is being or not to the decision center. The hard decision is easy to be implemented, and the occupied bandwidth for transmission is small. However, under the conditions of ensuring all the cooperative user strict synchronization, the detection performance of soft-decision is superior to that of hard decision, and the performance improvement is more obvious with the increase of the cooperative user's number 
[7]. The soft decision can provide more full, detailed, and effective information for the final decision, so in this paper the soft decision is used.

There are some classic fusion decision rules, such as “AND” rule, "OR” rule, "K” rank rule, selective combination(SC) rule, preferred sets combination rule, equal gain combination(EGC) rule, the maximum ratio combination rule, and so on[7]. In the above fusion decision rules, "OR" rules is simpler and more mature, so "OR" rule is used in this paper.

"OR" rule means that the decision center combines every node's local decision with logical "OR", that is, only one node determines that the authorized user is being, the decision center will claim that the authorized user is being.

Supposing the $i^{\text {th }}$ cognitive user's false alarm probability and detection probability are $p_{\mathrm{fi}}$ and $p_{\mathrm{d} i}$ respectively, the false alarm probability $Q_{\mathrm{f}}$ and detection probability $Q_{\mathrm{d}}$ of the system by "OR" rule are as follows.

$$
\begin{aligned}
& Q_{f}=1-\prod_{i=1}^{n}\left(1-p_{f i}\right) \\
& Q_{d}=1-\prod_{i=1}^{n}\left(1-p_{d i}\right)
\end{aligned}
$$

The missing detection probability is

$$
Q_{m}=1-Q_{d}
$$

\subsection{The Dual-threshold Energy Detection Model}

The energy detection contains single-threshold energy detection and dual-threshold energy detection. The threshold $\lambda$ of single threshold energy detection is not easy to be determined, and the accurate judgment cannot be made easily when the detected energy value is located round $\lambda$, so the dual-threshold energy detection method is adopted in this paper.

Two thresholds $\lambda_{\mathrm{L}}$ and $\lambda_{\mathrm{H}}$ are set in the traditional dual-threshold energy detection method. The primary user is determined in existence if the detected channel energy $E_{i}>\lambda_{\mathrm{H}}$, and presented with $H_{1}$. The primary user is determined not in existence if $E_{i}<\lambda_{\mathrm{L}}$, and presented with $H_{0}$. If $\lambda_{\mathrm{L}} E_{E} E_{i}<\lambda_{\mathrm{H}}$ the cognitive user sends the detected energy $E_{i}$ or nothing to the base station sends.

By simulation it is show that when $\lambda_{\mathrm{L}}<E_{i}<\lambda_{\mathrm{H}}$ and the cognitive user sends the detected energy $E_{i}$ to the base station, the simulation curve is almost same with the theoretical curve in AWGN channel. Therefore, the algorithm is improved based on the above rule.

\subsection{The Energy-cooperative Detection Model}

The improved decision rule based on the traditional dou- ble-threshold energy detection is as follows. The cognitive user $i$ sends the detected energy $E_{i}$ to the fusion decision center if $\lambda_{\mathrm{L}}<E_{i}<\lambda_{\mathrm{H}}$, and all these energy $E_{i}$ are collected in the fusion decision center. Then the average energy of these $E_{i}$ is calculated in the fusion decision center and marked as $\bar{E}$. Finally, compare $\bar{E}$ with $E_{i}$. The decision value of user $i$ is 1 if $\bar{E}<E_{i}$, which corresponds to $H_{1}$. The decision value of user $i$ is 0 if $\bar{E}>E_{i}$, which corresponds to $H_{0}$. In this way, the detection probability will be raised distinctly when there are a large number of users.

1) AWGN channel

In AWGN channel, let it mean the decision value come from user $\mathrm{i}$ and received by the base station. [8] Then

$$
\begin{gathered}
R_{i}=\left\{\begin{array}{cc}
E_{i}, & \lambda_{L}<E_{i}<\lambda_{H} \\
L_{i}, & \text { others }
\end{array}\right. \\
L_{i}=\left\{\begin{array}{cc}
0, & 0<E_{i}<\lambda_{L} \\
1, & E_{i}<\lambda_{H}
\end{array}\right.
\end{gathered}
$$

Suppose that there are $N$ cognitive users, $K$ of them send the local decision value to the base station, and the other $N-K$ cognitive users send the detected energy to the base station. The fusion decision center will make a decision according to the $N-K$ values. The decision rule is as follows.

$$
D=\left\{\begin{array}{l}
0, \quad 0 \leq \sum_{i=1}^{N-K} E_{i} \leq \lambda \\
1, \quad \sum_{i=1}^{N-K} E_{i}>\lambda
\end{array}\right.
$$

where, $\lambda$ is equivalent to the threshold of single threshold energy detection here, which is the average value of the $\lambda_{L}$ and $\lambda_{H} \cdot \sum_{i=1}^{N-K} E_{i}$ follows the distribution in formula (7).

$$
\sum_{i=1}^{N-K} E_{i} \sim \begin{cases}\chi_{2(N-K) u}^{2}, & H_{0} \\ \chi_{2(N-K) u\left(2 \gamma_{0}\right)}^{2}, & H_{1}\end{cases}
$$

where, $\gamma_{0}=\sum_{i=1}^{N-K} \gamma_{i}$ is the sum of the $N-K$ users' SNR. $u$ is the product of the time and the bandwidth, $u=\tau \cdot B$, $\tau$ is the period of the signal, and $B$ is the bandwidth of the signal. [9]

The fusion decision center will make a final decision according to the following formula [10].

$$
F=\left\{\begin{array}{rr}
1, & D+\sum_{i=1}^{K} L_{i}>1 \\
0, & \text { others }
\end{array}\right.
$$

Let $\Delta_{0, i}$ and $\Delta_{1, i}$ represent the probability that the 
detected energy value is between $\lambda_{L}$ and $\lambda_{H}$ for $H_{0}$ and $H_{1}$ respectively. Then the probability of detection, missing detection, and false alarm are as follows [11].

$$
\begin{aligned}
& p_{\mathrm{di} i}=P\left\{E_{i}>\lambda_{H} \mid H_{1}\right\}=Q_{u}\left(\sqrt{2 \gamma}, \sqrt{\lambda_{H}}\right) \\
& p_{\mathrm{fi}}=P\left\{E_{i}>\lambda_{H} \mid H_{0}\right\}=\frac{\Gamma\left(u, \lambda_{H} / 2\right)}{\Gamma(u)} \\
& p_{\mathrm{m} i}=P\left\{E_{i} \leq \lambda_{L} \mid H_{1}\right\}=1-\Delta_{1, i}-P_{\mathrm{d} i}
\end{aligned}
$$

where $Q_{u}$ is the normalized Marcumq function in $u$-order. $\Gamma(\cdot)$ and $\Gamma(\cdot, \cdot)$ are the complete and incomplete Gramma functions.

2) Rayleigh channel

In Rayleigh channel, the detection probability of double threshold for user $\mathrm{i}$ is similar to that of single threshold, just only using $\lambda_{H}$ to substitute $\lambda$.

\section{Simulation Analysis}

The energy-cooperative detection method is verified by simulation using Monte Carlo method. Supposing that all the parameters of the system are as follows: $u=5, N=5$, $\Delta_{0, i}=\Delta_{1, i}=0.01, \mathrm{SNR}=5$, the number of Monte Carlo simulation is 20000 .

1) AWGN channel

The simulation results of the improved method and the traditional method with "OR" rule are shown in Figure 1 in AWGN channel. It can be seen that the performance of the improved method is superior to that of the traditional method.

2) Rayleigh channel

The simulation results of the improved method and the traditional method with "OR" rule are shown in Figure 2 in Rayleigh channel. It can be seen that compared with the traditional method the improved method has no

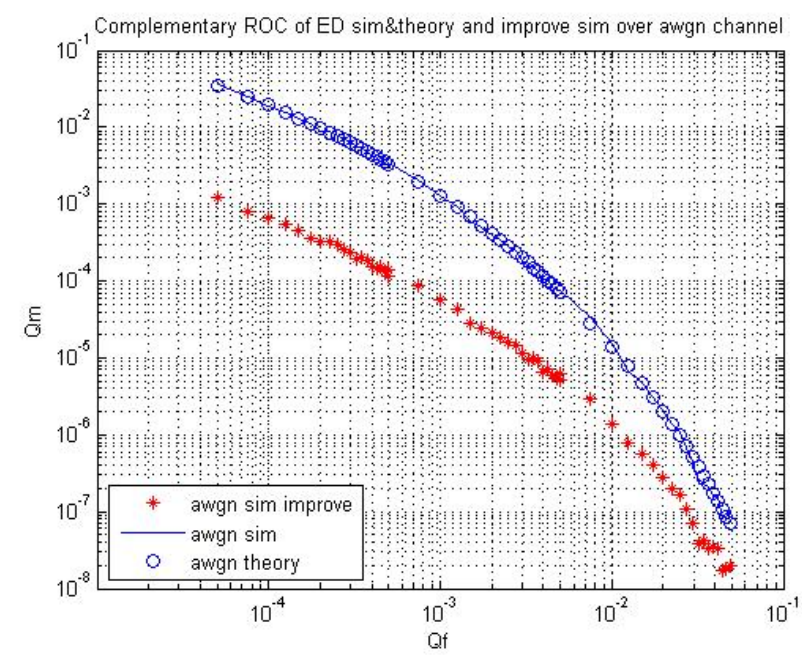

Figure 1. The performance comparison using "OR" rule in AWGN channel.

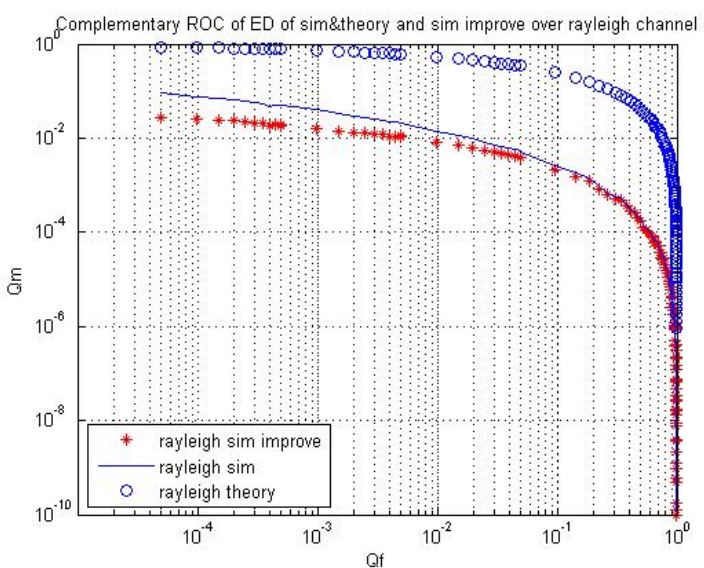

Figure 2. The performance comparison using “OR" rule in Rayleigh channel.

distinct improvement in performance. Only is $Q_{\mathrm{f}}$ low enough, the improved method is better. With $Q_{\mathrm{f}}$ increasing, the number of the energy value which is located between the two thresholds will be decreased rapidly. So the improved method is not effective in these conditions. That is why there is almost no performance improvement when $Q_{\mathrm{f}}$ is high.

\section{Conclusions}

Spectrum sensing is studied for multi-user cognitive radio networks in this paper. An improved method-Energy-cooperative detection method is developed, which combines the double threshold energy detecting method with the "OR" fusion decision rule. The advantages of the improved method are validated by simulation, especially in AWGN channel.

\section{Acknowledgements}

This work was supported by State Key Lab. of Power System, Tsinghua University; the Research Fund for joint China-Canada Research and Development Projects of The Ministry of Science and Technology, China (Grant No. 2010DFA11320); Beijing Municipal Science Technology Commission (Grant No. Z1111000665110 07); and the Youth Research and Innovation Program of the Electronic Engineering School, Beijing University of Posts and Telecommunications. (Grant No. 2012RC 0304 ).

\section{REFERENCES}

[1] A. S. Malik, O. Boyko, N. Atkar and W. F. Young, “A Comparative Study of MR Imaging Profile of Titanium Pedicle Screws,” Acta Radiologica, Vol. 42, No. 3, 2001, pp. 291-293. doi:10.1080/028418501127346846

[2] T. Hu and J. P. Desai, "Soft-Tissue Material Properties under Large Deformation: Strain Rate Effect,” Proceed- 
ings of the 26th Annual International Conference of the IEEE EMBS, San Francisco, 1-5 September 2004, pp. 2758-2761.

[3] P. Zhang and Z. Y. Feng, "The Cognitive Radio Networks,” Science press. 2010.

[4] T. Zhi, G. B. Giannakis, "Compressed Sensing for Wideband Cognitive Radios," Acoustics, Speech and Signal Processing. ICASSP, 2007, pp. 1357-1360.

[5] X. Z. Xie, "The Cognitive Radio Technology and Its Applications,” Electronics Industry Press. 2008.

[6] Harry URKOWITZ, "Energy Detection of Unknown Deterministic Signals.” Proceedings of the IEEE, Vol. 55, No. 40, 1967, pp. 523-531.

[7] F. F. Digham, Mohamed-Slim Alouini, Marvin K. Simon, "On the Energy Detection of Unknown Signals over Fading Channels," 03 IEEE International Conference on Communications, 2003, pp. 3575-3579.

[8] J. Jiang, X. Z. Tan and H. J. Sun, “Cooperative Algorithm for Cognitive Radio Networks Which is Based on Adaptive Election,” 2006 IEEE Region 10 Conference (TENCON, 2006, pp. 1-4.

[9] I. F. Akyildiz, W.Y. Lee, M. C. Vuran and V. Mohanty, "Next Generation/Dynamic Spectrum Access/Cognitive Radio Wireless Networks: A Survey," Computer Networks, 2006, Vol. 50, No. 13, pp. 2127-2159. doi:10.1016/j.comnet.2006.05.001

[10] G. Ganesan and Y. G. Li, “Agility Improvement Through Cooperation Diversity in Cognitive Radio,” Proceedings of IEEE Global Communication Conference. St Louis, Missouri, USA, Vol. 5, 2005, pp. 2505-2509.

[11] H. Z. Ma, Y. P. Liao and W. Jiang, "A Double-threshold Based Collaborative Spectrum Sensing Algorithm,” 2nd International Conference on Signal Processing Systems (ICSPS), 2010 , Vol. 3, pp. 729 - 732. M. Young. The Technical Writer' s Handbook. Mill Valley, CA: University Science, 1989. 